\title{
Multiple eruptive dermatofibromas occuring in a patient under hemodialysis
}

\author{
Sarra Ben Rejeb', Amen Dhaoui', Dorra Ben Ghachem¹, Asmahane Souissi², \\ Khadija Bellil ${ }^{1}$
}

${ }^{1}$ Department of Pathology, FSI Hospital, La Marsa, Tunisia, ${ }^{2}$ Department of Dermatology, FSI Hospital, La Marsa, Tunisia

Corresponding author: Dr. Sarra Ben Rejeb, E-mail: sarrabenrejeb88@yahoo.fr

\begin{abstract}
Dermatofibroma is a benign lesion of the skin, characterized by a fibroblastic proliferation. It is usually a solitary nodule, but some cases of patients diagnosed with multiple eruptive dermatofibromas have been reported. We herein describe another rare case of numerous ill-defined nodules occurring in a patient on long term-dialysis. Microscopic examination revealed typical features of dermatofibroma.
\end{abstract}

Key words: Multiple; Dermatofibroma; Pathology; Dermatology

\section{INTRODUCTION}

Dermatofibroma is a common benign skin tumor, most often occurring as a solitary lesion. Multiple eruptive dermatofibromas (MDF) have been reported is few cases. They are characterised by the presence of eruption reported within a short period of time of at least 15 lesions. This entity is commonly occurring on patients with underlying disease which is on more than $80 \%$ of cases an immune-mediated disease. In this report, we describe an additional case of multiple dermatofibromas.

\section{CASE REPORT}

A 70 year old woman presented with multiple (more than 15) yellowish and irregular papules and nodules developed within a period of 2 months (Fig. 1). They were distributed asymmetrically all over arms and thighs, ranging from 0.5 to $1 \mathrm{~cm}$ in diameter. She had medical history of chronic renal failure requiring regular dialysis since 10 years. There was no other systemic disease or any evidence of immunodeficiency.

Clinical examination revealed several firm, slightly raised yellowish nodules, ranging from 0.5 to $1 \mathrm{~cm}$ in diameter. The patient underwent excision-biopsy of a lesion of the arm.

Microscopic examination showed an epidermal hyperplasia with fibroblastic cell proliferation extending into the deep dermis. It was made of whorling fascicles of spindle cells with excessive collagen deposition (Figs. 2-4).

\section{Ethics}

This study was performed on human subjects; thus, all patients were aware of the presence of the study and they were fully informed about the drug and its side-effects.

\section{DISCUSSION}

Dermatofibroma (DF) is a benign fibrohistiocytic tumor which commonly occurs as a solitary lesion over lower extremities, more frequently in women than men. Few cases of multiple eruptive dermatofibromas have been reported accounting for less than $0.3 \%$ of all dermatofibromas.

Although the etiopathogeny of this entity is still unknown, they have been frequently reported in

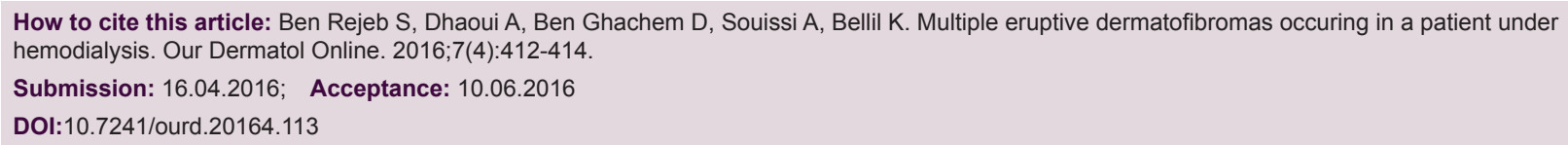




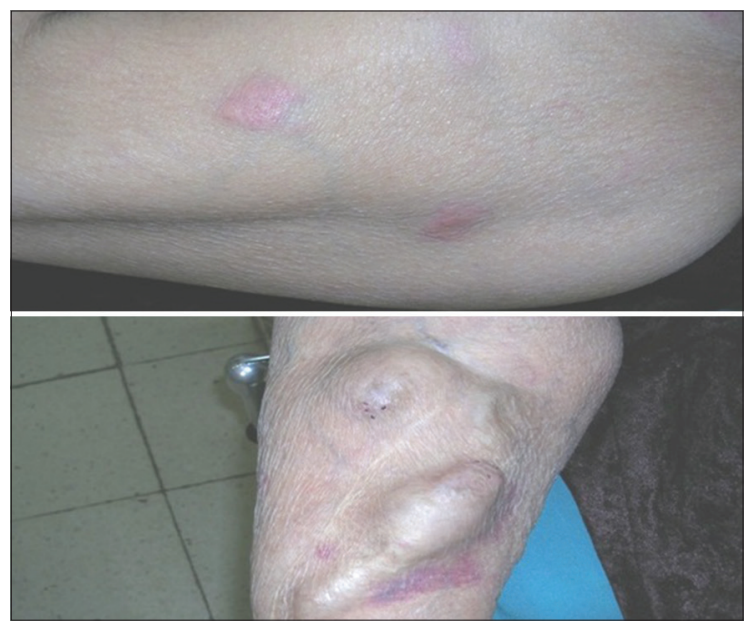

Figure 1: Fistula for hemodialysis associated to Several irregular red papules in the arm.

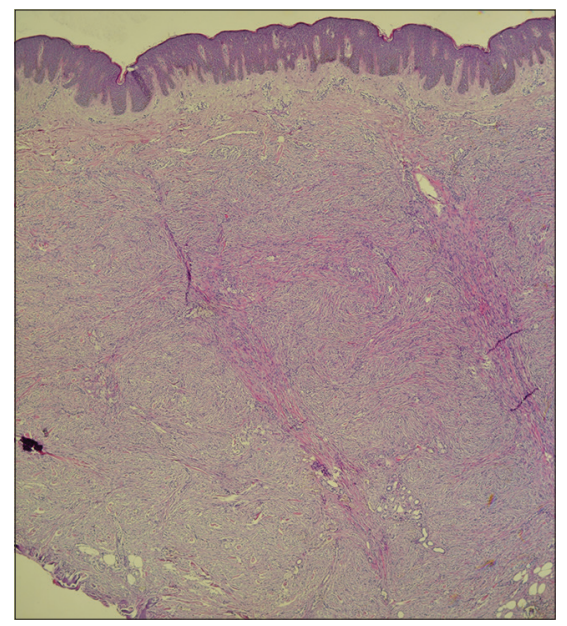

Figure 2: Epidermal hyperplasia with diffuse fibroblastic proliferation extendind in the deep dermis. (HE x 100).

association with autoimmune diseases or altered immune states, such as, in patients with HIV infection, lupus, chronic myelogenous leukemia, or organ transplant [1-4]. Aetiology of MDF is unknown. However, more than half of patients have underlying diseases, and more than $80 \%$ of the underlying diseases are immune mediated. MDF may be associated with autoimmune diseases or altered immune states, such as, in patients with HIV infection, lupus, dermatomyositis chronic myelogenous leukemia, or organ transplant. Also, Immunosuppressants drugs such as methotrexate and corticosteroids might cause MDF [5].

In this condition, multiple DF may be considered as a potential manifestation of an immune-mediated disease. Familial cases suggesting a genetic disorder have also been reported [6]. Our review of literature revealed that 22 of 72 subjects were otherwise healthy [7].

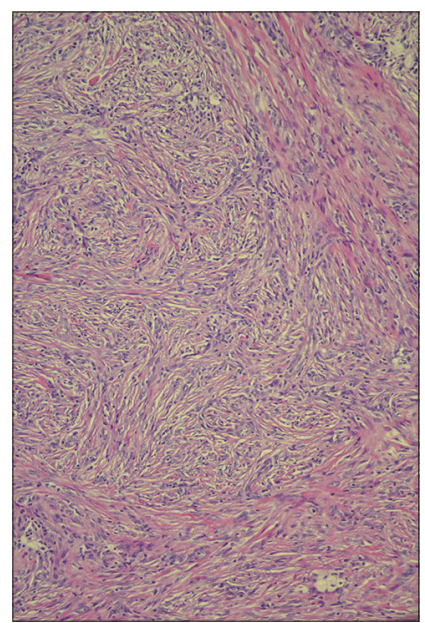

Figure 3: Fibroblastic proliferation showing storiform pattern. (HE x 200).

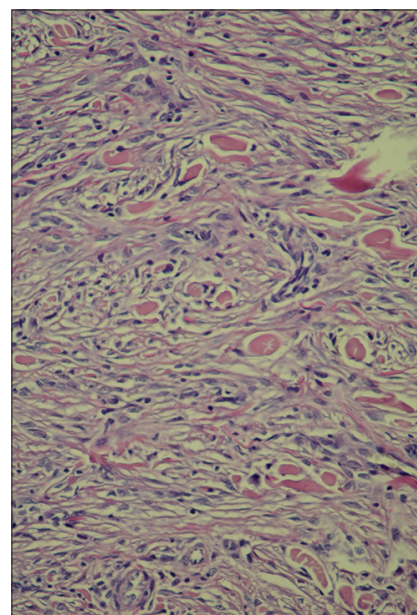

Figure 4: Fibroblastic proliferation mixed with bundles of collagen. (HE x 400).

In this report, we describe the case of multiple DF occurring in a patient on dialysis for chronic renal failure but without any evidence of other immunosuppressive condition.

To our knowledge, this association has not been reported previously.

Many authors have defined "multiple dermatofibromas" as the presence of at least 15 lesions appeared in a short period of time [8]. Given that incipient cases might be omitted, appearance of 5 to 8 dermatofibromas in 4 months has been proposed as sufficient to establish diagnosis. They are predominantly occurring on women and usually asymptomatic lesions, but itching has been reported in some cases. Eruptive dermatofibromas generally present characteristic pathologic features 
distinguishable from the sporadic cases. They are commonly showing a poorly circumscribed lesion epidermal hyperplasia, prominent bundles of collagen and a diffuse proliferation of fibroblastic cells. It may present a storiform pattern extending into the deep dermis and subcutaneous tissues.

\section{CONCLUSION}

Because of its frequent association to immunerelated disease, diagnosis of multiple eruptive DermatoFibroma should always lead us to rule out an underlying systemic diseases or the possibility of immunodeficiency of the patient. However, in our case, investigations didn't reveal any evidence of systemic disease or immune deficiency. Possibility of immune disorder due to dialysis may be considered.

\section{Consent}

The examination of the patient was conducted according to the Declaration of Helsinki principles. Written informed consent was obtained from the patient for publication of this article.

\section{REFERENCES}

1. Kanitakis J, Carbonnel E, Delmonte S, Livrozet JM, Faure M, Claudy A. Multiple eruptive dermatofibromas in a patient with HIV infection: case report and literature review. J Cutan Pathol. 2000;27:54-6.

2. Yamamoto T, Sumi K, Yokozeki H, Nishioka K. Multiple cutaneous fibrous histiocytomas in association with systemic lupus erythematosus. J Dermatol. 2005;32:645-9.

3. Alexandrescu DT, Wiernik PH. Multiple eruptive dermatofibromas occurring in a patient with chronic myelogenous leukemia. Arch Dermatol. 2005;141:397-8.

4. Kovach BT, Sams HH, Stasko T. Multiple atypical fibroxanthomas in a cardiac transplant recipient. Dermatol Surg. 2005;31:467-70.

5. Huang PY, Chu CY, Hsiao CH. Multiple eruptive dermatofibromas in a patient with dermatomyositis taking prednisolone and methotrexate. J Am Acad Dermatol. 2007; 57:S81-4.

6. Yazici AC, Baz K, Ikizoglu G, Koca A, Kokturk A, Apa DD. Familial eruptive dermatofibromas in atopic dermatitis. J Eur Acad Dermatol Venereol. 2006;20:90-2

7. Her Y, Ku SH, Kim KH. A case of multiple eruptive dermatofibromas in a healthy adult. Ann Dermatol. 2014;26:539-40.

8. Baraf CS, Shapiro L. Multiple histiocytomas: report of a case. Arch Dermatol. 1970;101:588-90.

Copyright by Sarra Ben Rejeb, et al. This is an open access article distributed under the terms of the Creative Commons Attribution License, which permits unrestricted use, distribution, and reproduction in any medium, provided the original author and source are credited.

Source of Support: Nil, Conflict of Interest: None declared. 